\title{
Design of Rhombus Shaped Fractal Antenna for WLAN and X-Band Applications
}

\author{
Shrikant Pai \\ Vidyalankar Institute of Technology, \\ Wadala, Mumbai
}

\author{
Pravin Patil \\ Vidyalankar Institute of Technology, \\ Wadala, Mumbai
}

\begin{abstract}
Low profile features of microstrip antennas attract many researchers to investigate the performance of these antennas in various ways.Generation of multiband characteristic takes place when an antenna is introduced with some geometrical distortions i.e. fractal which is then used to increase data rates of communication system. Generally, an antenna tends to resonate at a single frequency but fractal antenna is a special type which has multiple resonances. Fabrication of miniaturized antennas have become possible due to fractal technology. This letter presents the design of Rhombus shaped fractal antenna using Sierpinski gasket structure.Two Sierpinski gasket structures has been combined, one in an inverted fashion which ultimately leads to a rhombus shape and the performance of second iteration antenna is studied.A $50 \mathrm{ohms}$ microstrip line is used to excite the patch.Analysis and Simulation of the proposed antenna has been done with the help of HFSS 13.0,an electromagnetic simulation software. The proposed antenna has been designed to resonate for LTE (long term evolution), WLAN and X-band Applications.
\end{abstract}

\section{Keywords}

Fractal Technology, HFSS, Iterations, LTE, Multiband Antennas, Rhombus shape, Sierpinski Triangle, VSWR, WLAN

\section{INTRODUCTION}

The field of Antenna has attracted eyeballs of many researchers to explore diverse technologies in different ways. This field has gained significant demand with the advent of microstrip antennas. Antennas are considered to be the most important component or element in any wireless communication system.Microstrip patch antennas are basically known for their low profile(Antennas which are relatively thin and can be easily mounted onto a flat surface), small size, low weight and low cost.

Generally, all antennas tend to operate at single or dual frequency bands due to which different antennas are required for different applications which ultimately leads to limited space problems. In order to circumvent this problem, fractal antennas are put to use wherein a single antenna tends to operate at many frequency bands[1].Fractal Term was first coined by Benoitt Mandelbrott in the year 1975.By incorporating fractal shape onto the antenna geometry, multiband antennas can be constructed. Fractal shaped antennas tends to exhibit some interesting features due to their geometrical properties. Self similarity and space filling are the two significant properties exhibited by fractal antennas.Multiband behavior is the result of Self-similar structure of fractal antenna. Almost all wireless communication systems demand for compactness which can be achieved with the help of Space filling property of fractal antenna which increases antenna's electrical length and ultimately leads to antenna miniaturization.

Dr. Nathan Cohen assembled the first fractal antenna element to work on $2 \mathrm{~m}$ amateur band.He reported his initial work at an ARRL conventions in 1994 and published the first article on fractal antennas in 1995[1]-[4].Design of $2.45 \mathrm{GHz}$ triangular fractal antenna using fractal geometry has been simulated using Ansoft HFSS and was observed that increase in iterations led to improvement in VSWR and return loss[5].A planar fractal antenna for UHF RFID on metallic surface has been designed wherein a size reduction of $89.8 \%$ was achieved with respect to conventional rectangular patch[6].A bow- tie antenna with fractal property based on Sierpinski gasket has been simulated which demonstrated increase in bandwidth as compared to conventional bow-tie antenna[7].In[8] multiband fractal monopole antenna was first designed by Puente. In [9], design of multiband fractal antenna for cognitive radio applications was proposed wherein an E-shaped antenna covered GSM 1800, LTE and UMTS applications with good gain of about $5 \mathrm{~dB}$ and radiation efficiency of 90-95\% was achieved. In [10] design of crown shaped fractal antenna was proposed in which the antenna resonated at four different frequencies $8.4 \mathrm{GHz}$, $10.1 \mathrm{GHz}, 10.6 \mathrm{GHz}$ and $11.3 \mathrm{GHz}$ which is used for satellite, radar and wireless applications.

In the proposed work, two Sierpinski gasket antennas has been combined to form a rhombus shaped antenna and simulated with a view to resonate it for WLAN and X-band applications.HFSS 13.0 has been used for simulation purpose. The antenna has been simulated up to second iteration.Sierpinski gasket structures are basically known for their bandwidth, gain and efficiency making it an attractive candidate for Wi-fi,Wimax and other communication systems[7],[14].

\section{DESIGN APPROACH}

Figure 1 shows the geometry of basic Sierpinski gasket structure with first and second iteration which uses triangle as its base shape.Fig.2.(a),(b),(c) shows the geometry of initial, first and second iteration patch antenna respectively.

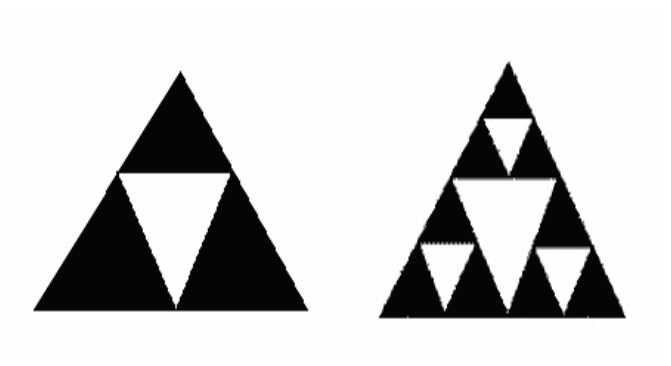

Fig 1: Basic Sierpinski gasket structure with first and second iteration 
The proposed antenna is the combination of two basic Sierpinski gasket structure[12], one in an inverted fashion which leads to rhombus shape. The patch has been proposed on RogersRT/Duroid substrate which is known for consistent performance at higher frequencies[13]. The substrate has thickness of $1.7 \mathrm{~mm}$ with dielectric constant $\boldsymbol{\epsilon}_{\boldsymbol{r}} 2.2$ and has dimensions of $75 \times 75 \mathrm{~mm}$. The design has been iterated twice.

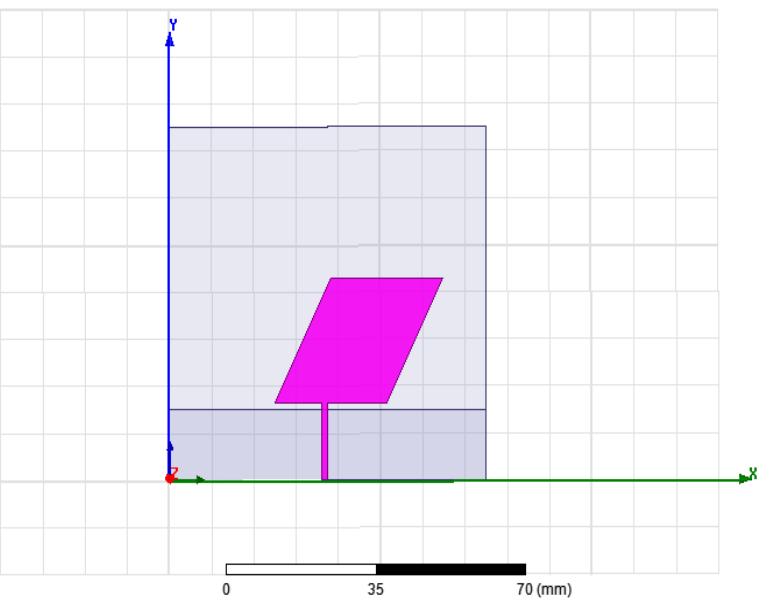

Fig 2(a): Initial iteration of the patch.

The operating resonant frequency of antenna depends on patch size, width of microstrip line, feed location and substrate thickness. So as to obtain better performance of antenna, these parameters should be optimized[11],[15]. All other essential parameters are summarized in table I.

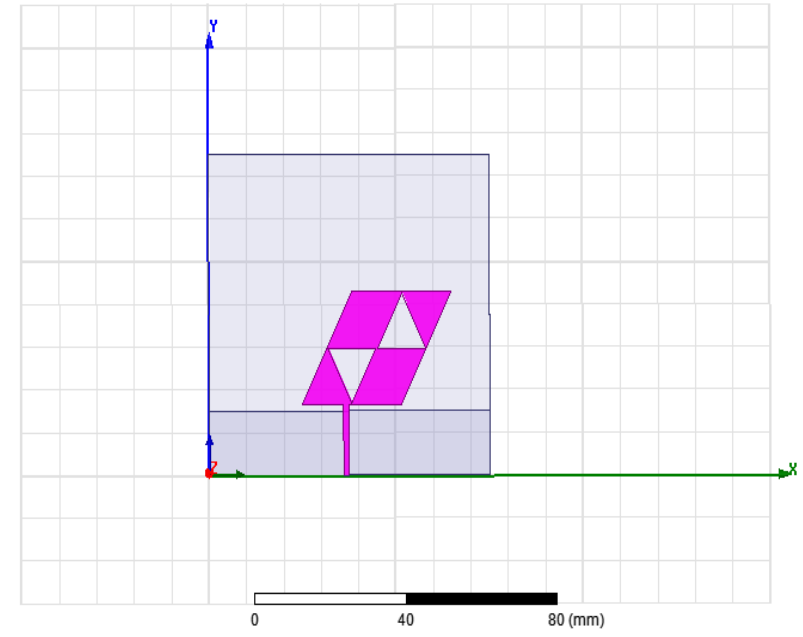

Fig 2(b): First iteration of the patch.

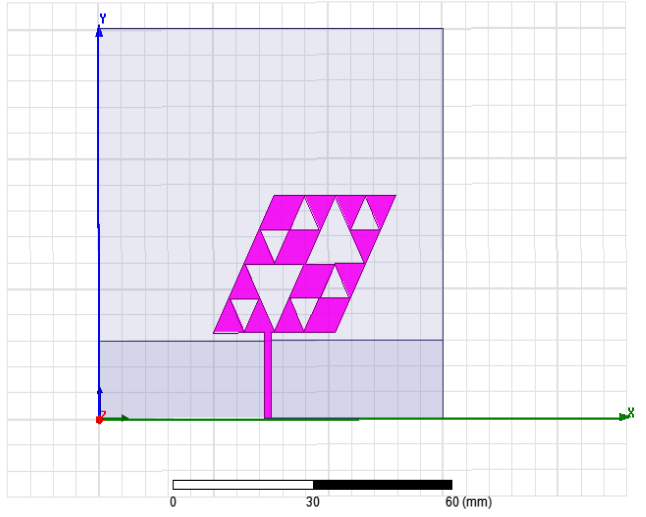

Fig 2(c): Second iteration of the patch.
Table 1: Important parameters with their values

\begin{tabular}{|c|c|}
\hline Parameters & Values in $\mathrm{mm}$ \\
\hline Feed location & $\mathrm{x}=0.98, \mathrm{y}=24, \mathrm{z}=0$ \\
\hline Length of feed & 16.47 \\
\hline Width of feed & 1.5 \\
\hline
\end{tabular}

\section{SIMULATION RESULTS}

Analysis and Simulation of proposed antenna is carried out with the help of Ansoft HFSS software.VSWR, gain and return loss were obtained which indicates that proposed antenna is most suitable for wireless applications.Fig. 3 shows the simulated results of second iteration return loss. The characteristic of the proposed rhombus shaped antenna at second iteration is summarized in table II.

Table 2: Characteristics of proposed antenna at second iteration

\begin{tabular}{|c|c|c|c|}
\hline Frequency $(\mathrm{GHz})$ & $\begin{array}{c}\text { Return } \\
\text { loss(dB) }\end{array}$ & VSWR & Bandwidth \\
\hline 2.7 & -17.25 & 1.31 & $300 \mathrm{Mhz}$ \\
\hline 5.7 & -11.95 & 1.67 & $500 \mathrm{Mhz}$ \\
\hline 9.4 & -22.03 & 1.17 & $1 \mathrm{GHz}$ \\
\hline
\end{tabular}

Triple resonance is achieved at $2.7 \mathrm{GHz}, 5.7 \mathrm{GHz}$ and $9.4 \mathrm{GHz}$ with impedance bandwidths of $300 \mathrm{MHz}, 500 \mathrm{MHz}$ and $1 \mathrm{GHz}$ respectively. Impedance bandwidth is range of frequencies below -10dB.Fig. 4 indicates the corresponding VSWR of second iteration at desired frequencies wherein it is well within the accepted range i.e. below 2 for all desired frequencies. A good gain of about 3.9 and $4.2 \mathrm{~dB}$ is achieved at first and second iteration respectively. The gain achieved has almost omnidirectional characteristics as shown in Fig.5.(a),(b).The proposed antenna has an axial ratio of $6 \mathrm{~dB}$ indicating circular polarization which has better ability to reject reflected waves[11].

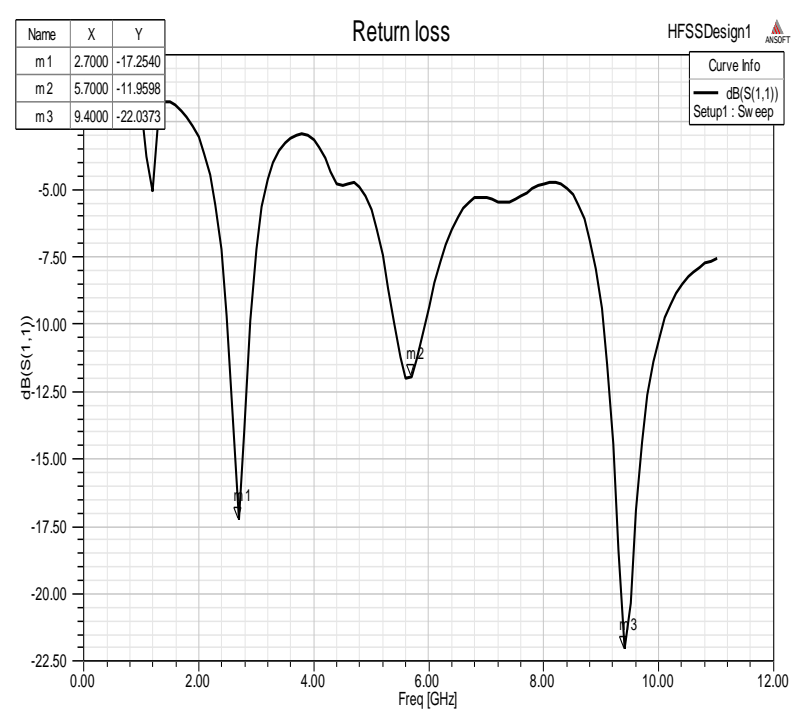

Fig 3: Return loss of Second iteration 


\section{CONCLUSION}

Fractal antenna using combination of Sierpinski gasket geometry was successfully designed and simulated. The design is simulated with the help of HFSS tool. The proposed antenna is built on partial ground plane and fed through microstrip line. The antenna resonated at three frequencies namely $2.7 \mathrm{GHz}, 5.7 \mathrm{GHz}$ and $9.4 \mathrm{GHz}$ which are desired frequencies.It was observed that the gain of antenna improves with higher order iterations. The desired resonant frequency covers LTE $(2.5-2.7 \mathrm{GHz})$, WLAN $(5.7 \mathrm{GHz})$ and X-band $(8-$ $12 \mathrm{GHz}$ ) applications. The future work is to fabricate and test the performance of the proposed antenna so as to validate the simulated results.

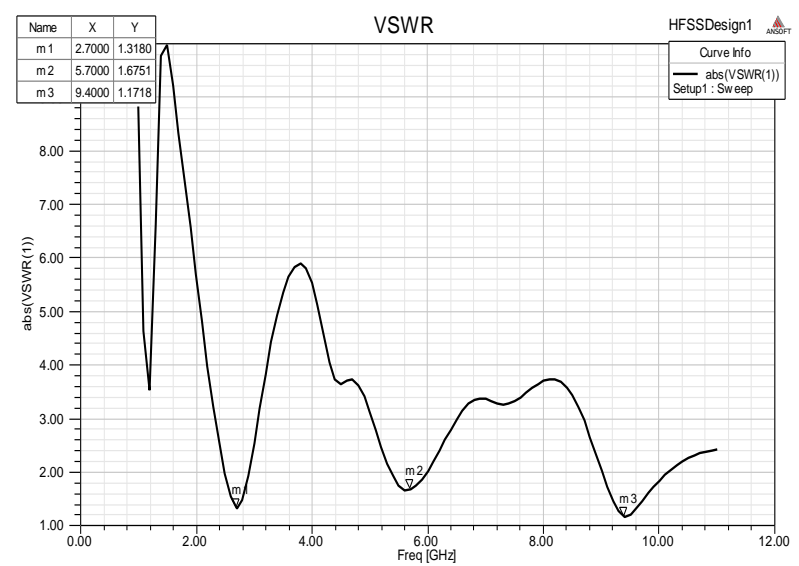

Fig 4: VSWR of Second iteration
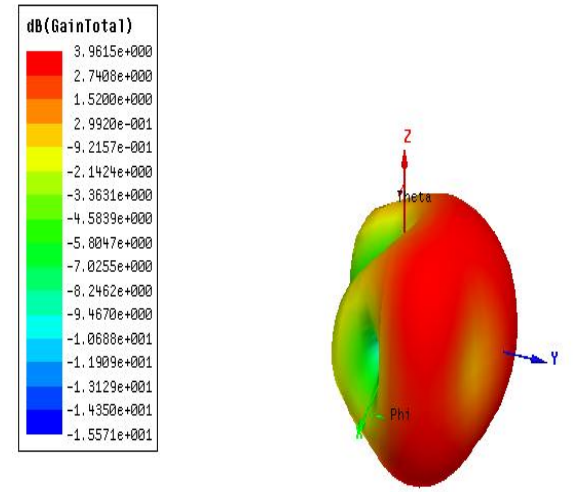

Fig 5(a): Gain of First iteration patch antenna
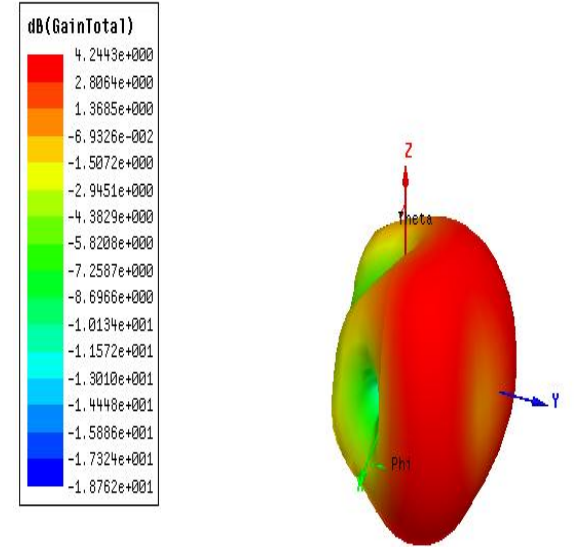

Fig 5(b): Gain of Second iteration patch antenna

\section{ACKNOWLEDGMENTS}

Any project work that has achieved its objective is solely due to cumulative efforts taken by everyone in that project. So, I would like to thank my project guide, Prof.Pravin.Patil for continuous encouragement, invaluable supervision and inspired guidance offered.

\section{REFERENCES}

[1] N.Cohen,"Fractal antennas,"Communication Quaterly, summer, 1994, pp.7-22.

[2] N.Cohen and R.G.Hulled, "Fractal loops and the small loop approximations,"Communication Quaterly, winter, 1996, pp.77-81.

[3] N.Cohen,"Fractal and Shaped Dipoles,"Communication Quaterly, summer, 1996, pp.25-36.

[4] N.Cohen,"Fractalantennas:Part2,"Communication Quaterly,Summer,1996,pp.7-22.

[5] R.LYadava, M.Ram and S.Das, "Multiband Triangular fractal antenna for Mobile communications," International Journal of Engineering Science and Technology, Vol.2 (11), 2010, 6335-6348.

[6] G.Monti, L.Catarinucci, L.Tarricone, "Compact Micro strip antenna for RFID Applications,"Progress in Electromagnetic Research Letters,Vol.8,191-199,2009.

[7] Samira Ahmed, Arun Kumar Singh, Devesh Kumar, "Design of a Sierpinski Fractal Shaped Bow-Tie Antenna for WLAN Applications," International Journal of Scientific and Engineering Research, Volume 5, Issue 5, May-2014.

[8] C.Puente, J.Romeo, "Fractal multiband antennas based on Sierpinski Gasket,"IEEE Electronics letter, 32, 1 Jan 1996, pp.1-2.

[9] Peshal.B.Nayak, Sudhanshu Verma, Preetam Kumar, "Multiband fractal antenna design for Cognitive Radio Applications,"IEEE Antennas and Propagation Society, 2013.

[10] Sachin Chauhan, Jitendra Kr.Deegwal, Devendra Soni, Prashant Singodia, "A Design of Crown Shape Fractal Antenna,"International Journal of Engineering and Innovative Technology, Vol2, Issue 3, 2012.

[11] G.Kumar and K.P.Ray, "Broadband microstrip antennas,"Artect House, Norwood, Mass, USA 2003.

[12] Pandian S.Siva Sundara, Suriyakala C.D.,"A Novel Multiband Sierpinski triangular fractal antenna for Cognitive Radio," International Conference on Circuits, Power and Computing Technologies, 2013, vol., no., pp.803, 807, 20-21 March 2013.

[13] P.W.Tang, P.F. Wahid,"Hexagonal fractal antenna,'IEEE Antennas and Wireless Propagation Letters, vol.3, no.1, pp.111-112, Dec.2004.

[14] H.O.Peitgen, H.Jurgens and D.Saupe,"Chaos and Fractals: New Frontiers in Science,"New York :SpringerVerlag .1992.

[15] Constantine.A.Balanis, “Antenna Theory, Analysis and Design,"John Wiley and Sons Inc. $2^{\text {nd }}$ edition, 1997. 